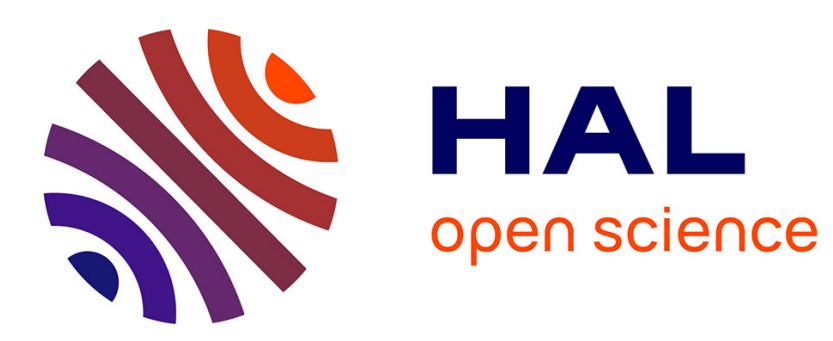

\title{
Modélisation stochastique d'écoulements diphasiques avec changement de phase
}

\author{
Olivier Hurisse, Jean-Pierre Minier
}

\section{To cite this version:}

Olivier Hurisse, Jean-Pierre Minier. Modélisation stochastique d'écoulements diphasiques avec changement de phase. Comptes Rendus Mécanique, 2011, 339 (6), pp.418-431. 10.1016/j.crme.2011.04.004 . hal-01587685

\section{HAL Id: hal-01587685 \\ https://hal.science/hal-01587685}

Submitted on 14 Sep 2017

HAL is a multi-disciplinary open access archive for the deposit and dissemination of scientific research documents, whether they are published or not. The documents may come from teaching and research institutions in France or abroad, or from public or private research centers.
L'archive ouverte pluridisciplinaire HAL, est destinée au dépôt et à la diffusion de documents scientifiques de niveau recherche, publiés ou non, émanant des établissements d'enseignement et de recherche français ou étrangers, des laboratoires publics ou privés. 


\title{
Modélisation stochastique d'écoulements diphasiques avec changement de phase
}

\author{
Olivier Hurisse ${ }^{a}$ Jean-Pierre Minier ${ }^{a}$ \\ ${ }^{a}$ EDF Rध̈D, Dépt. MFEE, 6 quai Watier, 78400 Chatou, France.
}

\begin{abstract}
Résumé
La modélisation stochastique avec point de vue lagrangien a déjà été développée et appliquée au cadre des écoulements monophasiques et des écoulements diphasiques incompressibles. Cet article propose une extension de ce formalisme aux écoulements diphasiques compressibles avec changement de phase (de type eau-vapeur par exemple). L'accent est mis sur deux aspects essentiels, dont la formulation est nouvelle en modélisation stochastique : un modèle de changement de phase et l'expression d'une contrainte portant sur la conservation du volume. Enfin, à titre d'exemple, des éléments de réflexion sont présentés pour deux modèles bifluides.
\end{abstract}

\begin{abstract}
Stochastic modelling of two-phase flows including phase change. Stochastic modelling has already been developped and applied for single-phase flows and incompressible two-phase flows. In this article, we propose an extension of this modelling approach to two-phase flows including phase change (e.g. for steam-water flows). Two aspects are emphasised: a stochastic model accounting for phase transition and a modelling constraint which arises from volume conservation. To illustrate the whole approach, some remarks are eventually proposed for two-fluid models.
\end{abstract}

Key words: Fluid mechanics; two-phase flow; stochastic process; phase change; compressible; bubble

Mots-clés : Mécanique des fluides ; écoulement diphasique ; processus stochastique ; changement de phase ; compressible ; bulle

\section{Abridged English version}

Over the last decades, modelling of compressible two-phase flows has been tackled using approaches based on sets of Partial Differential Equations (PDEs), whose unknowns are eulerian mean fields [1,2]. Therefore, the derivation of constitutive laws, even those describing highly non-linear local phenomena

Email addresses: olivier.hurisse@edf.fr (Olivier Hurisse), jean-pierre.minier@edf.fr (Jean-Pierre Minier). 
(e.g. the phase change), are directly done using mean fields, which represent a restricted statistical information. These eulerian models are satisfactory for a certain range of industrial situations. Nonetheless, when the governing phenomena are highly non-linear (e.g. when dealing with cavitation, polydispersed bubbles, nucleate boiling ...), such approaches can lead to descriptions that are not accurate enough. In these situations, one can benefit of the stochastic modelling approach using a lagrangian point of view $[3,4]$ (or lagrangian stochastic modelling). This approach has already been applied to different configurations: incompressible single-phase flows have been widely addressed, see [3] among others for a complete presentation, whereas the effect of compressibility in single-phase flows has been less investigated [5]. Modelling of two-phase flows introduces new aspects, some of which have been addressed in [4] for incompressible flows. In the present paper, we propose an extension to compressible two-phase flows. It is worth recalling that the lagrangian stochastic approach amounts to closing the Probabiliy Density Function (PDF) of the variables chosen to describe the flow, from which classical PDEs for the corresponding mean fields may be obtained.

Flows are described using particles, where each particle represents a sufficient number of molecules so that the classical notions of the continuum mechanics still make sense. Each particle of phase $k$ (with $k=1,2$ ) is associated with a mass $m_{k}$, a position in the physical space $x_{k}$, a velocity $u_{k}$, a volume $v_{k}$ and an internal energy $e_{k}$. This set of variables is one possible minimal set of variables necessary to describe non-uniform transient compressible flows (in the compressible single-phase case depicted in [5], the pressure has been chosen instead of the volume). All other quantities are functions of these five variables: for example pressures $p_{k}=\mathcal{P}_{k}\left(v_{k}, e_{k}\right)$ and the densities $\rho_{k}=m_{k} / v_{k}$. An important point to be put forward is that, since the position in space $x_{k}$ and the velocity $u_{k}$ (and thus the trajectory) are variable, we are dealing with a lagrangian description. The time evolution of the variables is governed by stochastic differential equations, each one involving two modelling terms: one term that describes the evolution of the expectation, and one term defining the behavour of the dispersion around the expectation. These terms are not detailled in the sequel, except those for the volumes $v_{k}$.

Phase change is modelled using two ingredients: a condition to fulfill for each particle to change phase and an instantaneous "transformation" applied to the variables $\left(m_{k}, x_{k}, u_{k}, v_{k}, e_{k}\right)$ when the transition occurs. The choice for the latter is straightforward: the value of the variables does not change. Despite its simplicity, this choice obviously guarantees conservation of the variables $\left(m_{k}, x_{k}, u_{k}, v_{k}, e_{k}\right)$ and of the total local number of particles. Phase transition is assumed to be instantaneous. In order to account for the non-zero characteristic time scale $\tau$ for the phase change of a set of identical particles, we add a variable $\mathcal{D}$ to each particle. This variable follows an exponential law with a parameter $1 / \tau$. It represents the lifetime of the particle before the phase transition. Finally, phase change occurs if and only if it is followed by a decrease of the chemical potential and if the realization $D$ of $\mathcal{D}$ is less than $\delta t$, where the latter stands for the physical time represented by the phase transition (i.e. for example, $\delta t$ is a time step when considering a numerical scheme).

Once the model has been defined, it is possible to write a Chapmann-Kolmogorov equation (2) which governs the time evolution of the PDF $f^{L}\left(t ; Z_{1}^{L}, Z_{2}^{L}\right)$ of the process in the phase-space, where $Z_{k}^{L}=$ $\left(x_{k}^{*}, u_{k}^{*}, m_{k}^{*}, v_{k}^{*}, e_{k}^{*}\right)$ for $k=1,2$. This PDF is the basis of the definition of the volumic fraction for the phase $k$ :

$$
\alpha_{k}(t, x) \stackrel{\text { def }}{=} \int v_{k}^{*} \delta\left(x-x_{k}^{*}\right) f^{L}\left(t ; Z_{1}^{L}, Z_{2}^{L}\right) d Z_{1}^{L} d Z_{2}^{L} .
$$

This eulerian field represents the fraction of volume per unit of volume occupied by the phase $k$. In order to ensure the consistency of our model with the physics, the sum of the volumic fractions must always be equal to one. This is related to the so-called volume conservation property. The Chapmann-Kolmogorov equation for $f^{L}$ leads to PDEs (3) on the expectation of any function of $Z_{k}^{L}$. Using the PDEs (3) for the expectation of the inverse of the densities, two PDEs for $\alpha_{k}$ may be found. Then, it can be shown that the constraint $\alpha_{1}(t, x)+\alpha_{2}(t, x)=1$ is equivalent to the constraint (5) on the modeling terms $A_{k}^{v}$ for 
the volumes of the particles. When this constraint is fulfiled, it appears that all the PDE systems on the mean fields that can be derived from the stochastic model contain the two equations (6).

It is interesting to focus on the two equations (6) for dispersed spherical bubbles in a liquid. On the one hand, a simplified Rayleigh-Plesset equation (8) for the pulsation of the bubbles can be used to define $A_{1}^{v}$. Then the modelling term $A_{2}^{v}$ is chosen according to (5), to ensure the property of volume conservation. The resulting equation for $\alpha_{1}$ is close to the equation of the models of $[7,8,9,10]$. On the other hand, a modelling term for the bubble volume that allows to retrieve the standard two-fluid model can be exhibited (12). It is very intricate and its exact physical content remains an open question.

The modelling approach proposed herein suggests some forthcoming works. First, systems of PDEs can be derived from a physical description of compressible two-phase flows, carried out by a lagrangian stochastic model. In this approach, the physical hypotheses underlying PDE systems appear more clearly. At last, numerical simulations of two-phase flows involving phase transition could be improved by applying hybrid schemes, following the ideas developped in $[12,13]$. The latter requires to have a stochastic model and a PDEs system derived from this model.

\section{Introduction}

La physique des écoulements diphasiques compressibles est complexe et, à ce jour, certains de ses aspects restent encore mal compris. C'est notamment le cas des échanges de masse, de quantité de mouvement ou d'énergie entre les phases qui constituent des éléments clefs dans la phénoménologie des applications industrielles.

Depuis plusieurs dizaines d'années la modélisation mathématique de tels écoulements repose essentiellement sur des approches basées sur des champs eulériens moyens. Les systèmes d'équations aux dérivées partielles (EDP) associés à ces modèles sont obtenus en appliquant des opérateurs de moyenne sur des "poches de fluide" monophasiques [1,2]. D’un point de vue formel, une fonction indicatrice définit la répartition spatio-temporelle des phases et dans chacune des zones ainsi décrites un système d'EDP monophasique (Euler par exemple en compressible) régit l'évolution instantanée des champs du fluide. Les systèmes d'EDP pour les champs moyens sont ensuite obtenus par application d'un opérateur de moyenne. Finalement, la modélisation des échanges entre les phases est réalisée a posteriori sur les champs moyens obtenus.

Les approches basées sur une description particulaire avec modélisation stochastique permettent d'établir les modèles sur les grandeurs instantanées et non sur les champs moyens [3,4]. Ensuite, une fois le modèle instantané établi, il est possible d'en déduire un modèle basé sur un système EDP pour les champs moyens. Ce dernier n'est pas équivalent à la description instantanée, mais il constitue une extraction d'une partie de son information. On peut donc dériver un système d'EDP sur des champs moyens à partir d'un modèle particulaire stochastique.

Il est important de noter que les deux approches sont complémentaires. Suivant la physique que l'on veut modéliser et suivant l'information que l'on cherche à obtenir, l'une ou l'autre des approches est préférable. L'atout majeur de la modélisation particulaire stochastique repose dans sa capacité à traiter sans approximation les phénomènes locaux non-linéaires comme la convection, les réactions chimiques, etc ...

Le travail présenté constitue une extension aux écoulements diphasiques compressibles d'un formalisme de modélisation particulaire stochastique qui est déjà appliqué aux écoulements monophasiques réactifs $[3,5]$ (compressibles et incompressibles) ainsi qu'aux écoulements diphasiques incompressibles de type fluide-particules [4]. En effet, ce formalisme est tout particulièrement adapté à la modélisation des écoulements diphasiques compressibles, car il permet notamment de traiter de façon naturelle le change- 
ment de phase et la convection. Par ailleurs, il permet d'expliciter le lien entre une description lagrangienne stochastique et un modèle d'EDP portant sur des champs moyens.

Dans ce qui suit, on se propose tout d'abord d'écrire un modèle particulaire stochastique simple prenant en compte le changement de phase. On présente ensuite les outils permettant d'obtenir un modèle eulérien sur les champs moyens. L'accent est mis sur la formulation d'une contrainte de conservation du volume qui n'est pas explicitement abordée dans [4]. Ce dernier point s'avère essentiel, d'une part car il assure la consistance physique du modèle, mais aussi parce que son étude permet d'ouvrir des perspectives sur les types de modèles sur champs moyens que l'on peut déduire d'une telle approche. Ce lien est illustré dans la section 5 à l'aide de modèles existants. Dans la section 5.1, le comportement du volume d'une bulle est décrit grâce à une loi de type Rayleigh-Plesset simplifiée, puis une EDP décrivant le comportement moyen des bulles est déduite de la formulation instantanée.

\section{Le modèle stochastique lagrangien}

Le principe de la description particulaire du fluide est de représenter l'écoulement par un ensemble de particules discrètes. Les particules sont des objets mathématiques ponctuels auxquels sont attachées les variables permettant de décrire l'écoulement : volume, pression, vitesse... Chaque particule représente un ensemble de molécules suffisamment grand pour que les notions classiques de mécanique des milieux continus aient un sens, notamment les grandeurs thermodynamiques. Si la position dans l'espace est vue comme une variable attachée aux particules, la trajectoire de ces dernières devient elle-même une variable. On parle alors de description particulaire lagrangienne, par opposition aux descriptions eulériennes pour lesquelles la position en espace est un paramètre.

L'évolution en temps des variables de chaque particule est régie par un système d'équations différentielles en temps (et non en espace puisque la position n'est pas un paramètre mais une variable). Parmi les phénomènes participant à la description de cette évolution, certains peuvent être modélisés de manière stochastique, ce qui reflète le manque d'information associé au choix de la description particulaire (probabilités à une particule ou à $\mathrm{N}$ particules, voir [4]). L'évolution en temps est alors déterminée par un système d'équations différentielles stochastiques (EDS).

Le modèle que l'on se propose d'écrire repose sur ces deux principes : description particulaire lagrangienne et modélisation stochastique, ce que l'on désigne par modélisation lagrangienne stochastique.

Le point de départ de cette construction est le choix des variables de description des écoulements que l'on souhaite modéliser. En pratique, le jeu de variables peut être différent pour les deux phases, mais on se contentera de prendre le même ensemble de variables pour les deux phases. Puis, une fois le jeu de variables choisi, le système d'EDS pour chaque phase sera décrit. La construction du modèle est achevée par la description de la prise en compte du changement de phase, qui est traité de façon particulière.

Pour conserver des équations les plus compactes possibles, on se référera à l'indice $k=1$ pour la phase 1 et $k=2$ pour la phase 2 . Ainsi lorsque l'on s'intéresee à la phase d'indice $k$, l'indice $3-k$ désigne l'autre phase : si $k=1$ (resp. $k=2$ ), alors $3-k=2$ (resp. $3-k=1$ ). De plus, les sections de présentation $\mathrm{du}$ formalisme et du modèle sont écrites dans un cadre mono-dimensionnel, c'est-à-dire que les positions et les vitesses sont scalaires et que les volumes sont homogènes à des distances. L'extension à l'espace tri-dimensionnel est triviale et la dernière section est écrite dans ce cadre. Par ailleurs, il est possible d'enrichir la modélisation proposée en introduisant de nombreuses variables, mais on se limite dans la suite aux seules variables essentielles pour décrire des écoulements diphasiques compressibles. 


\subsection{Le choix des variables de description}

Le choix des variables est essentiel car il détermine les phénomènes qui peuvent être pris en compte dans la modélisation et ceux qui ne le peuvent pas. On se limite ici aux variables indispensables à la description des écoulements diphasiques compressibles. Chaque particule de phase $k$, et de masse $m_{k}$, porte donc une information sur sa propre trajectoire grâce à une position $x_{k}$ et à une vitesse $u_{k}$. Leur thermodynamique est, quant à elle, décrite par un volume $v_{k}$ et une énergie interne $e_{k}$. Pour chaque phase le jeu de variables est donc: $m_{k}, x_{k}, u_{k}, v_{k}$ et $e_{k}$.

Toute autre grandeur associée à la particule doit s'exprimer en fonction de ce jeu de variables. C'est notamment le cas de la pression et de la densité de la particule $: p_{k}=\mathcal{P}_{k}\left(v_{k}, e_{k}\right)$ et $\rho_{k}=m_{k} / v_{k} ;$ où $\mathcal{P}_{k}$ est une fonction donnée (loi d'état). Le choix de définir deux pressions différentes pour les deux phases s'explique par la volonté de pouvoir prendre en compte les variations de volume associés aux écarts de pression entre phases, comme dans le cas des équations de type Rayleigh-Plesset. Ce point sera éclairé dans la section 5 .

La notion de phase vue a été introduite dans [4] pour la modélisation de la vitesse. Elle repose sur le principe que les trajectoires des particules des deux phases sont différentes : on définit pour la phase 1 la vitesse $u_{1}^{s, 2}$ qui est la vitesse de phase 2 vue par une particule de phase 1 se déplaçant à vitesse $u_{1}$. Par souci de simplicité, de telles variables ne sont pas définies ici. Néanmoins, les résultats présentés dans le reste de ce document restent valides avec ou sans ces variables supplémentaires.

\subsection{Le choix des équations d'évolution}

Il y a deux manières d'écrire une EDS sur une variable $\Phi$. La forme intégrale :

$$
\Phi(t)-\Phi\left(t_{0}\right)=\int_{t_{0}}^{t} A d t+\int_{t_{0}}^{t} B d w_{t},
$$

est la plus correcte d'un point de vue mathématique, mais on utilisera la notation $d \Phi=A d t+B d w_{t}$ qui a l'avantage de la concision. Dans ces deux équations, $w_{t}$ désigne un processus stochastique de Wiener et la deuxième intégrale du terme de droite est définie au sens de Ito [6]. Les termes $A$ et $B$ modélisent respectivement les phénomènes lents et rapides.

Le système décrivant l'évolution en temps du jeu de variables des particules est donc composé de cinq EDS. La première assure la constance de la masse pour chacune des particules : $d m_{k}=0$. En fait, les masses des particules peuvent être différentes, mais chaque particule garde la même masse au cours du temps. Les deuxième et troisième équations définissent la trajectoire en espace des particules :

$$
d x_{k}=u_{k} d t, d u_{k}=A_{k}^{u} d t+B_{k}^{u} d w_{k}^{u} .
$$

Enfin, les deux dernières équations portent sur la thermodynamique :

$$
d v_{k}=A_{k}^{v} d t+B_{k}^{v} d w_{k}^{v}, d e_{k}=A_{k}^{e} d t+B_{k}^{e} d w_{k}^{e} .
$$

Cette dernière équation exprimera le premier principe de la thermodynamique. L'ensemble des termes de modélisation $A_{k}^{u}, A_{k}^{e}, B_{k}^{u}$ et $B_{k}^{e}$ ne sera pas explicité dans ce document, en revanche des fermetures seront proposées à titre d'exemple pour $A_{k}^{v}$ et $B_{k}^{v}$.

\subsection{Prise en compte du changement de phase}

La modélisation du changement de phase avec une approche lagrangienne stochastique est fondamentalement différente de celles proposées pour les approches classiques par EDP sur des champs moyens, où 
elle est souvent identifiée avec le transfert de masse. Dans l'approche adoptée ici, le changement de phase n'est pas pris en compte en faisant varier la masse de chaque particule (d'où la forme de l'équation de masse définie dans la section 2.2), mais en modifiant le nombre de particules de chaque phase.

La transition de phase d'une particule est considérée comme instantanée. La prise en compte du temps caractéristique $\tau$ de transition de phase pour un ensemble de particules est décrite un peu plus bas dans cette section, elle est associée à une variable aléatoire $\mathcal{D}$ représentant une durée de vie. Deux ingrédients sont alors nécessaires pour définir le changement de phase : (i) une règle définissant les nouvelles valeurs des variables de la particule après la transition et (ii) une condition à remplir pour que la transition de phase soit effective.

Lorsqu'une particule change de phase, les valeurs des différentes variables sont égales aux valeurs correspondantes aux même variables pour la particule avant la transition. En d'autres termes, si une particule de phase $k$ associée aux variables $\left(m_{k}, x_{k}, u_{k}, v_{k}, e_{k}\right)$ change de phase pour devenir une particule de phase $3-k$, alors on définit :

$$
m_{3-k}=m_{k}, \quad x_{3-k}=x_{k}, \quad u_{3-k}=u_{k}, \quad v_{3-k}=v_{k}, \quad e_{3-k}=e_{k} .
$$

Si on attribuait une couleur différente pour chacune des phases, la définition ci-dessus revient à dire que lorsqu'elle change de phase la particule change juste de couleur. L'avantage de cette définition est que l'on assure naturellement la conservation de la masse du mélange, du volume du mélange, de la quantité de mouvement du mélange et de l'énergie du mélange.

Remarque. Il est possible de faire d'autres choix concernant les grandeurs conservées lors de la transition de phase. Il est par exemple envisageable de conserver la pression au lieu du volume :

$$
\mathcal{P}_{3-k}\left(v_{3-k}, e_{3-k}\right)=\mathcal{P}_{k}\left(v_{k}, e_{k}\right) .
$$

Néanmoins, le choix qui est fait ici de conserver le volume permet d'avoir une expression simple de la notion de conservation du volume du mélange présentée dans la section 4 .

Pour compléter la définition du changement de phase, une condition doit être spécifiée. Classiquement, en modélisation thermodynamique, un volume de fluide change de phase si et seulement si ce changement s'accompagne d'une diminution du potentiel chimique associé. Si $\mu_{k}\left(v_{k}, e_{k}\right)$ désigne ce potentiel chimique, et si $\left(v_{k}, e_{k}\right)$ et $\left(v_{3-k}, e_{3-k}\right)$ sont les grandeurs thermodynamiques associées à la particule respectivement avant et après la transition de phase, la condition ci-dessus se traduit par le respect de l'inégalité :

$$
\mu_{k}\left(v_{k}, e_{k}\right)>\mu_{3-k}\left(v_{3-k}, e_{3-k}\right) .
$$

En fait, cette inégalité traduit le caractère irreversible de la transition de phase pour des variables de descriptions données.

Par définition la transition de phase est considérée comme instantanée pour les particules prises individuellement. Afin que le temps caractéristique associé au changement de phase d'un ensemble de particules ne soit pas nul, une condition supplémentaire est nécessaire. On suppose donc que la transition de phase instantanée représente une durée physique $\delta T$. Par exemple pour une simulation numérique, cette durée sera le pas de temps de discrétisation. On introduit une variable aléatoire $\mathcal{D}$ pour chaque particule. Celleci suit une loi exponentielle de paramètre $1 / \tau$, où $\tau$ est le temps caractéristique de transition de phase pour un ensemble de particules. La condition supplémentaire de transition de phase est que la réalisation $D$ de $\mathcal{D}$ soit inférieure à $\delta T$, soit : $D<\delta T$. En d'autres termes, cette variable $D$ modélise la "durée de vie" qu'il reste à la particule avant qu'elle ne change de phase. Si cette durée est supérieure au temps physique représenté par la transition de phase, alors la particule n'a pas le temps de changer de phase. Ainsi, le temps caractéristique de changement de phase d'un ensemble de particules est $\tau$. Au final, la condition de changement de phase est donc :

$$
\mu_{k}\left(v_{k}, e_{k}\right)>\mu_{3-k}\left(v_{3-k}, e_{3-k}\right) \quad \text { et } \quad D<\delta T \text {. }
$$


La transition de phase telle qu'elle est décrite ci-dessus correspond à un mécanisme très simple. Il consiste à modifier uniquement la phase à laquelle la particule appartient, les grandeurs qui lui sont attachées restant inchangées. Par ailleurs, il est essentiel de noter que ce modèle de changement de phase est indépendant des modèles associés aux équations d'évolution des particules (section 2.2). Cette distinction est flagrante lorsque l'on considère l'équation de Chapmann-Kolmogorov (section 3.2) : les termes de modélisation associés aux EDS correspondent à des termes de dérivées d'ordre 1 dans l'espace des phases, tandis que le modèle de changement de phase est présent en tant que terme source.

\section{Description dans l'espace des phases}

Le modèle de la section précédente utilise une description basée sur des processus stochastiques. Il est donc naturel d'y associer une fonction densité de probabilité (FDP) dont les arguments sont : le temps et les variables de description. Cette FDP est régie dans l'espace des phases (i.e. l'espace constitué par l'ensemble des valeurs possibles pour les variables de description) par une équation de type ChapmannKolmogorov [6]. Il est alors possible de décrire le comportement de champs moyens de fonctions des variables de description.

\subsection{Quelques définitions sur l'espace des phases}

Pour toute variable, son image dans l'espace des phases sera notée à l'aide d'une étoile. On définit sur cet espace deux vecteurs pour chaque phase : le premier correspondant à un point de vue lagrangien $Z_{k}^{L}=\left(x_{k}^{*}, u_{k}^{*}, m_{k}^{*}, v_{k}^{*}, e_{k}^{*}\right)$ et le second à un point de vue eulérien $Z_{k}^{E}=\left(u_{k}^{*}, m_{k}^{*}, v_{k}^{*}, e_{k}^{*}\right)$. On notera par la suite $Z^{L}=\left(Z_{1}^{L}, Z_{2}^{L}\right)$. La FDP lagrangienne jointe notée $f^{L}$ a pour arguments le paramètre temps $t$ et les vecteurs de variables lagrangiens $Z_{k}^{L}$. La quantité $f^{L}\left(t ; Z^{L}\right) d Z^{L}$ représente alors la probabilité de trouver le processus stochastique $\left(x_{1}, u_{1}, m_{1}, v_{1}, e_{1}, x_{2}, u_{2}, m_{2}, v_{2}, e_{2}\right)$ dans un voisinage $d Z^{L}$ autour de l'état $Z^{L}$ à l'instant $t$. On notera que cette quantité est sans dimension, et donc $f^{L}$ est homogène à l'inverse de $Z^{L}$. L'intégration de $f^{L}$ sur l'ensemble de l'espace des phases donne naturellement 1 à chaque instant $t$ :

$$
\int f^{L}\left(t ; Z^{L}\right) d Z^{L}=1
$$

et la moyenne de toute fonction $H\left(Z^{L}\right)$ de la variable $Z^{L}$ contre $f^{L}$ s'écrit alors :

$$
<H\left(Z^{L}\right)>^{L}(t) \stackrel{\text { def }}{=} \int H\left(Z^{L}\right) f^{L}\left(t ; Z^{L}\right) d Z^{L} .
$$

A partir de cette FDP jointe, on définit deux fonctions de densité marginales eulériennes associées à chacune des phases :

$$
f_{k}^{E}\left(t, x ; Z_{k}^{E}\right) \stackrel{\text { def }}{=} \int \delta\left(x-x_{k}^{*}\right) f_{k}^{L}\left(t ; Z_{k}^{L}\right) d x_{k}^{*}, \quad k=1,2,
$$

où $\delta($.$) désigne la fonction de Dirac. Ces fonctions de densité ne sont plus des FDP car leur intégrale sur$ l'espace des phases ne donne pas toujours 1. Une conséquence de la restriction en espace associée à la fonction de Dirac est que la position devient un paramètre et non plus une variable : avec la définition de $f_{k}^{E}$, on adopte le point de vue eulérien. On définit ensuite la fraction volumique $\alpha_{k}$ qui dépend du temps et de l'espace (en tant que paramètre) :

$$
\alpha_{k}(t, x) \stackrel{\text { def }}{=} \int v_{k}^{*} f_{k}^{E}\left(t, x ; Z_{k}^{E}\right) d Z_{k}^{E}
$$

Cette grandeur définit la proportion de volume occupée par la phase $k$ en $x$ à l'instant $t$. La fonction de Dirac est homogène à l'inverse d'un volume, en conséquence un rapide calcul permet de remarquer que 
$\alpha_{k}$ est sans dimension. Par ailleurs, les volumes $v_{k}^{*}$ étant strictement positifs, $\alpha_{k}$ est positive et s'annule si statistiquement aucune particule de phase $k$ ne se trouve en $x$ à l'instant $t$. La fraction volumique a une grande importance dans le cadre des écoulements diphasiques, car elle assure en partie le couplage entre les phases (ce point est abordé dans la section 4).

Pour toute grandeur $H_{k}\left(Z_{k}^{E}\right)$ ne dépendant que de $Z_{k}^{E}$, et donc ne dépendant pas de la position de la particule, on définit deux grandeurs moyennes $\left\langle H_{k}\right\rangle_{k}^{\mathcal{R}}$ (qui correspond, dans l'esprit, à la moyenne de Reynolds) et $\left\langle H_{k}\right\rangle_{k}^{\mathcal{F}}$ (qui correspond, dans l'esprit, à la moyenne de Favre), qui dépendent de la position $x$ et du temps $t$ considérés :

$$
\alpha_{k}(t, x)\left\langle H_{k}\right\rangle_{k}^{\mathcal{R}}(t, x) \stackrel{\text { def }}{=} \int v_{k}^{*} H_{k}\left(Z_{k}^{E}\right) f_{k}^{E}\left(t, x ; Z_{k}^{E}\right) d Z_{k}^{E},
$$

et

$$
\alpha_{k}(t, x)\left\langle\rho_{k}\right\rangle_{k}^{\mathcal{R}}(t, x)\left\langle H_{k}\right\rangle_{k}^{\mathcal{F}}(t, x) \stackrel{\text { def }}{=} \int m_{k}^{*} H_{k}\left(Z_{k}^{E}\right) f_{k}^{E}\left(t, x ; Z_{k}^{E}\right) d Z_{k}^{E} .
$$

où on rappelle que le masse peut s'écrire $m_{k}^{*}=v_{k}^{*} \rho_{k}\left(Z_{k}^{E}\right)$. Ces deux définitions n'ont de sens que si la fraction volumique n'est pas nulle, c'est-à-dire si statistiquement il y a au moins une particule en $x$ à l'instant $t$. Par la suite, les arguments $(t, x)$ seront souvent omis par souci de concision.

La moyenne $\langle.\rangle_{k}^{\mathcal{R}}$ correspond à une moyenne d'ensemble, tandis que la moyenne $\langle.\rangle_{k}^{\mathcal{F}}$ définit une moyenne pondérée par la masse volumique de phase $k$. Cet aspect est mis en lumière lorsqu'on explicite les deux relations ci-dessous entre ces deux moyennes. D'une part, la définition de $\langle.\rangle_{k}^{\mathcal{F}}$ appliquée à $1 / \rho_{k}\left(Z_{k}^{E}\right)$ fournit une relation de consistance (1), qui est utile pour la suite :

$$
\left\langle\frac{1}{\rho_{k}\left(Z_{k}^{E}\right)}\right\rangle_{k}^{\mathcal{F}}=\frac{1}{\left\langle\rho_{k}\left(Z_{k}^{E}\right)\right\rangle_{k}^{\mathcal{R}}}
$$

D'autre part, elle permet d'expliciter une relation entre les deux moyennes qui est indépendante des fractions volumiques :

$$
\left\langle H_{k}\left(Z_{k}^{E}\right)\right\rangle_{k}^{\mathcal{F}}=\frac{\left\langle\rho_{k}\left(Z_{k}^{E}\right) H_{k}\left(Z_{k}^{E}\right)\right\rangle_{k}^{\mathcal{R}}}{\left\langle\rho_{k}\left(Z_{k}^{E}\right)\right\rangle_{k}^{\mathcal{R}}} .
$$

Enfin, on introduit la notion d'espérance conditionnelle de la variable $A$ sachant que la variable $B$ est égale à $b$, qui est valable pour les moyennes $\langle\cdot\rangle_{k}^{\mathcal{R}}$ et $\langle\cdot\rangle_{k}^{\mathcal{F}}$. C'est une fonction de $b$ notée $\left\langle A \mid B^{*}=b\right\rangle$ ou encore de manière courte $\langle A \mid B\rangle$. Si $(a, b) \rightarrow f_{A, B}(a, b)$ désigne la FDP jointe pour $A$ et $B$, et si $b \rightarrow f_{B}(b)=\int f_{A, B}(a, b) d a$ est la FDP marginale de $B$, alors on a la définition suivante :

$$
<A \mid B^{*}=b>\stackrel{\text { def }}{=} \int a^{*} f_{A \mid B}\left(a^{*}, b\right) d a^{*} \quad \text { avec } \quad f_{A \mid B}(a, b)=\frac{f_{A, B}(a, b)}{f_{B}(b)} .
$$

Cette définition conduit à la relation :

$$
<A \mid B^{*}=b>=<A \delta\left(B^{*}-b\right)>\left(f_{B}(b)\right)^{-1} .
$$

\subsection{L'équation de Chapmann-Kolmogorov}

Si le changement de phase n'est pas pris en compte dans notre modélisation, les résultats classiques en terme de processus stochastiques sans sauts montrent que l'évolution dans l'espace des phases de la FDP $f^{L}$ est régie par une équation de Fokker-Planck [6]. Or le changement de phase tel qu'il est décrit dans la section 2.3 correspond à un processus de saut. En conséquence, l'évolution de $f^{L}$ est décrite par une équation de Chapmann-Kolmogorov [6], qui est plus générale et prend en compte les sauts dans les processus. 
On définit la mesure de probabilité de saut $W\left(Z^{L^{\prime}} \mid t ; Z^{L}\right)$ pour un couple de particules composé d'une particule de chaque phase. Cette mesure est définie pour $Z^{L} \neq Z^{L^{\prime}}$, elle représente la densité de probabilité par unité de temps de sauter instantanément en $t$ de l'état $Z^{L}$ à l'état $Z^{L^{\prime}}$. Conformément au modèle décrit à la section 2.3 , celle-ci s'écrit :

$$
\begin{array}{r}
W\left(Z_{1}^{L^{\prime}}, Z_{2}^{L^{\prime}} \mid t ; Z_{1}^{L}, Z_{2}^{L}\right)=\frac{1}{\tau} \delta\left(Z_{1}^{L^{\prime}}-Z_{2}^{L}\right) \delta\left(Z_{1}^{L}-Z_{2}^{L^{\prime}}\right) \mathcal{H}(\delta t-D) \\
\mathcal{H}\left(\mu_{1}\left(Z_{1}^{L}\right)-\mu_{2}\left(Z_{2}^{L^{\prime}}\right)\right) \mathcal{H}\left(\mu_{2}\left(Z_{2}^{L}\right)-\mu_{1}\left(Z_{1}^{L^{\prime}}\right)\right),
\end{array}
$$

où $\mathcal{H}$ désigne la fonction de Heaviside. L'équation de Chapmann-Kolmogorov associée à $f^{L}$ est alors :

$$
\begin{aligned}
\frac{\partial}{\partial t}\left(f^{L}\left(t ; Z^{L}\right)\right)= & \sum_{k=1,2}\left\{\sum _ { Y _ { k } \in \{ x _ { k } ^ { * } , u _ { k } ^ { * } , m _ { k } ^ { * } , v _ { k } ^ { * } , e _ { k } ^ { * } \} } \left(-\frac{\partial}{\partial Y_{k}}\left(<A_{k}^{Y} \mid Z^{L}>^{L} f^{L}\right)\right.\right. \\
& \left.\left.+\frac{\partial^{2}}{\partial Y_{k}^{2}}\left(<\left(B_{k}^{Y}\right)^{2} / 2 \mid Z^{L}>^{L} f^{L}\right)\right)\right\} \\
& +\int W\left(Z^{L} \mid t ; z^{L}\right) f^{L}\left(t ; z^{L}\right) d z^{L}-\int W\left(z^{L} \mid t ; Z^{L}\right) f^{L}\left(t ; Z^{L}\right) d z^{L} .
\end{aligned}
$$

En multipliant cette équation par $\delta\left(x-x_{k}^{*}\right)$ et en intégrant sur $Z_{3-k}^{L}$ et $x_{k}^{*}$, on peut déduire de l'équation ci-dessus une équation pour les densités eulériennes $f_{k}^{E}$. Cette dernière permet d'obtenir une équation sur les champs moyens.

\subsection{L'équation sur les champs moyens}

Si on multiplie l'équation sur $f_{k}^{E}$ par $m_{k}^{*} H\left(Z_{k}^{E}\right)$ puis qu'on intègre sur $Z_{k}^{E}$, on obtient une équation aux dérivées partielles en temps et espace portant sur la moyenne $\left\langle H\left(Z_{k}^{E}\right)\right\rangle_{k}^{\mathcal{F}}$ de $H\left(Z_{k}^{E}\right)$ :

$$
\begin{array}{r}
\frac{\partial}{\partial t}\left(\alpha_{k}\left\langle\rho_{k}\right\rangle_{k}^{\mathcal{R}}\left\langle H_{k}\right\rangle_{k}^{\mathcal{F}}\right)+\frac{\partial}{\partial x}\left(\alpha_{k}\left\langle\rho_{k}\right\rangle_{k}^{\mathcal{R}}\left\langle u_{k} H_{k}\right\rangle_{k}^{\mathcal{F}}\right)=\int m_{k}^{*} H_{k}\left(Z_{k}^{E}\right) \delta\left(x-x_{k}^{*}\right) \mathcal{T}_{k}\left(t ; Z_{k}^{L}\right) d Z_{k}^{L} \\
+\sum_{Y_{k} \in\left\{u_{k}^{*}, m_{k}^{*}, v_{k}^{*}, e_{k}^{*}\right\}} \alpha_{k}\left\langle\rho_{k}\right\rangle_{k}^{\mathcal{R}}\left(\left\langle A_{k}^{Y} \frac{\partial}{\partial Y_{k}}\left(H_{k}\left(Z_{k}^{E}\right)\right) \mid Z_{k}^{E}\right\rangle_{k}^{\mathcal{F}}+\left\langle\left(B_{k}^{Y}\right)^{2} / 2 \frac{\partial^{2}}{\partial Y_{k}^{2}}\left(H_{k}\left(Z_{k}^{E}\right)\right) \mid Z_{k}^{E}\right\rangle_{k}^{\mathcal{F}}\right) .
\end{array}
$$

Le terme $\mathcal{T}_{k}\left(t ; Z_{k}^{L}\right)$ qui apparait au second membre de l'équation regroupe les différentes contributions (sous forme d'intégrales) portant sur la probabilité de saut $W\left(Z^{L^{\prime}} \mid t ; Z^{L}\right)$ :

$$
\mathcal{T}_{k}\left(t ; Z_{k}^{L}\right)=\int\left\{\int W\left(Z^{L} \mid t ; z^{L}\right) f^{L}\left(t ; z^{L}\right) d z^{L}-\int W\left(z^{L} \mid t ; Z^{L}\right) f^{L}\left(t ; Z^{L}\right) d z^{L}\right\} d Z_{3-k}^{L} .
$$

Les termes associés au changement de phase dans (3) satisfont la propriété de conservation suivante.

Propriété. Soit une fonction $H(u, m, v, e)$ telle que $H_{1}\left(Z_{1}^{E}\right)=H\left(Z_{1}^{E}\right)$ et $H_{2}\left(Z_{2}^{E}\right)=H\left(Z_{2}^{E}\right)$, alors :

$$
\int m_{1}^{*} H_{1}\left(Z_{1}^{E}\right) \delta\left(x-x_{1}^{*}\right) \mathcal{T}_{1}\left(t ; Z_{1}^{L}\right) d Z_{1}^{L}+\int m_{2}^{*} H_{2}\left(Z_{2}^{E}\right) \delta\left(x-x_{2}^{*}\right) \mathcal{T}_{2}\left(t ; Z_{2}^{L}\right) d Z_{2}^{L}=0 .
$$

La démonstration de cette propriété découle directement de la forme de $W$. Elle est fondamentale car elle assure que lors de la transition de phase, la moyenne de toute quantité $H$ est conservée pour le mélange. Par exemple, l'application de cette propriété pour la masse, $H(u, m, v, e)=1$, permet de s'assurer que la masse moyenne du mélange est conservée. Pour $H(u, m, v, e)=u$ on obtient la conservation du moment moyen du mélange et pour $H(u, m, v, e)=e$ on obtient la conservation de l'énergie interne moyenne du mélange. 
L'équation (3) permet de décrire les variations en temps et en espace (donc avec un point de vue eulérien) des moyennes de n'importe quelle fonction dépendant des vitesse, masse, volume ou énergie interne des particules d'une phase. Par conséquent, il s'agit d'une extraction d'une partie des données contenues dans le système d'EDS définissant l'écoulement. A ce titre, les équations sur les champs moyens contiennent moins d'information que les EDS.

Pour $H_{k}\left(Z_{k}^{E}\right)=1$, l'équation (3) fournit les équations de masse moyennes :

$$
\frac{\partial}{\partial t}\left(\alpha_{k}\left\langle\rho_{k}\right\rangle_{k}^{\mathcal{R}}\right)+\frac{\partial}{\partial x}\left(\alpha_{k}\left\langle\rho_{k}\right\rangle_{k}^{\mathcal{R}}\left\langle u_{k}\right\rangle_{k}^{\mathcal{F}}\right)=\int m_{k}^{*} \delta\left(x-x_{k}^{*}\right) \mathcal{T}_{k}\left(t ; Z_{k}^{L}\right) d Z_{k}^{L}
$$

dont la forme est classique. Il est important de remarquer que ces équations sont indépendantes des termes de modélisation $A_{k}^{\phi}$. Par ailleurs, le modèle de changement de phase proposé dans la section 2.3 assure naturellement la conservation de la masse totale en $(t, x)$. Grâce à la propriété énoncée ci-dessus, la somme sur les deux phases des termes sources de (4), associés au changement de phase, est nulle. En choisissant $H_{k}\left(Z_{k}^{E}\right)=u_{k}^{*}$ et $H_{k}\left(Z_{k}^{E}\right)=e_{k}^{*}$, on peut obtenir respectivement les équations portant sur la quantité de mouvement moyenne et sur les énergies internes moyennes.

Bien que cela ne soit pas explicité ici, il est possible d'obtenir pour chaque phase une équation aux dérivées partielles en temps et espace portant sur la corrélation entre n'importe quelle fonction des variables de description $Z_{k}^{E}$.

\section{La contrainte de conservation du volume}

Dans la section 3, la fraction volumique $\alpha_{k}$ a été définie en se basant sur le volume $v_{k}$. Ces grandeurs assurent en fait un lien fort entre les deux phases, qui jusqu'à maintenant sont traitées de manière relativement indépendante. Le but de cette section est double. Tout d'abord la notion de conservation du volume sera explicitée, puis il en sera déduit des contraintes portant sur les termes de modélisation des volumes $A_{1}^{v}$ et $A_{2}^{v}$.

\subsection{La notion de conservation du volume}

Le point de départ de cette section est la ré-écriture de la somme des fractions volumiques définies à la section 3.1. En effet, celle-ci peut s'écrire sous la forme d'une unique intégrale :

$$
\alpha_{1}(t, x)+\alpha_{2}(t, x)=\int\left(v_{1}^{*} \delta\left(x-x_{1}^{*}\right)+v_{2}^{*} \delta\left(x-x_{2}^{*}\right)\right) f^{L}\left(t ; Z^{L}\right) d Z^{L} .
$$

Cette somme représente le volume statistiquement occupé par unité de volume par l'ensemble des particules en $x$ à l'instant $t$. C'est sur cette grandeur que repose l'expression de la conservation du volume, elle se traduit par la propriété suivante.

Propriété. En chaque point $x$ et à chaque instant $t$, la somme des fractions de volume des particules des phases par unité de volume doit être égale à 1 . Autrement dit :

$$
\forall(t, x), \quad \alpha_{1}(t, x)+\alpha_{2}(t, x)=1 .
$$

Cette propriété exprime le fait que les particules statistiquement présentes en $x$ à l'instant $t$ doivent occuper tout le volume qui est à leur disposition. Il s'agit en fait d'assurer la consistance du modèle constitué de deux phases avec la physique. Pour ce faire, la manière la plus évidente est de traduire cette propriété en une contrainte portant sur la modélisation des volumes (i.e. sur les termes $A_{k}^{v}$ ). 


\subsection{Contrainte de conservation du volume}

La propriété de conservation du volume telle qu'elle est exprimée dans la section 4.1 porte sur le mélange des phases et fait intervenir les fractions volumiques, qui sont des grandeurs moyennes associées aux volumes des particules. Pour que cette propriété soit respectée, une contrainte sur les termes de modélisation des volumes $A_{k}^{v}$ doit être assurée.

En utilisant la relation $(1)$, pour $H_{k}\left(Z_{k}^{E}\right)=1 / \rho_{k}\left(Z_{k}^{E}\right)$ l'équation de la section 3.3 donne l'équation suivante :

$$
\frac{\partial}{\partial t}\left(\alpha_{k}\right)+\frac{\partial}{\partial x}\left(\alpha_{k}\left\langle u_{k}\right\rangle_{k}^{\mathcal{R}}\right)=\alpha_{k}\left\langle\frac{A_{k}^{v}}{v_{k}} \mid Z_{k}^{E}\right\rangle_{k}^{\mathcal{R}}+\int v_{k}^{*} \delta\left(x-x_{k}^{*}\right) \mathcal{T}_{k}\left(t ; Z_{k}^{L}\right) d Z_{k}^{L}
$$

Lorsqu'on somme ces deux équations, les termes associés au changement de phase s'annulent. En effet, lorsqu'une particule change de phase elle conserve son volume et sa position, ainsi le volume global est conservé en tout point lors de la transition de phase.

On suppose que l'initialisation à $t=0$ de l'écoulement est telle que $\alpha_{1}(0, x)+\alpha_{2}(0, x)=1$. En sommant pour les deux phases l'équation ci-dessus, il apparaît que la propriété de conservation du volume sera respectée si et seulement si la contrainte suivante est satisfaite :

$$
\frac{\partial}{\partial x}\left(\alpha_{1}\left\langle u_{1}\right\rangle_{1}^{\mathcal{R}}+\alpha_{2}\left\langle u_{2}\right\rangle_{2}^{\mathcal{R}}\right)=\alpha_{1}\left\langle\frac{A_{1}^{v}}{v_{1}} \mid Z_{1}^{E}\right\rangle_{1}^{\mathcal{R}}+\alpha_{2}\left\langle\frac{A_{2}^{v}}{v_{2}} \mid Z_{2}^{E}\right\rangle_{2}^{\mathcal{R}} .
$$

En posant $\alpha_{2}=0$, et donc $\alpha_{1}=1$, dans la contrainte (5), celle-ci redonne la contrainte de conservation telle qu'elle est classiquement écrite pour les écoulements monophasiques.

En définitive, si l'on veut assurer la conservation du volume, la modélisation des volumes des particules de chaque phase ne peut être faite de manière totalement indépendante. On peut ainsi énoncer l'équivalence suivante dans le cas où la somme des fractions volumiques est initialement égale à 1 à $t=0$ : la somme des fractions volumiques vaut 1 pour tout $(t, x)$ si et seulement si la relation (5) est vraie pour tout $(t, x)$.

Si les termes de modélisation des volumes respectent la contrainte $(5)$ et si $\alpha_{1}(0, x)+\alpha_{2}(0, x)=1$, alors le sous-système formé des deux équations aux dérivées partielles sur les fractions volumiques est équivalent au sous-système suivant :

$$
\left\{\begin{aligned}
& \alpha_{1}(t, x)+\alpha_{2}(t, x)=1, \\
& \frac{\partial}{\partial t}\left(\alpha_{1}\right)+\frac{\partial}{\partial x}\left(\alpha_{1}\left\langle u_{1}\right\rangle_{1}^{\mathcal{R}}\right)=\alpha_{1}\left\langle\frac{A_{1}^{v}}{v_{1}} \mid Z_{1}^{E}\right\rangle_{1}^{\mathcal{R}}+\int v_{1}^{*} \delta\left(x-x_{1}^{*}\right) \mathcal{T}_{1}\left(t ; Z_{1}^{L}\right) d Z_{1}^{L} .
\end{aligned}\right.
$$

Ce sous-système est un élément clef des modèles de champs eulériens décrivant les écoulements diphasiques, que les phases soient compressibles ou pas. Il intervient dans le couplage entre les phases. Comme on le verra dans la section suivante, il peut être présent soit sous forme explicite soit sous forme implicite.

Remarque. Dans le cas de deux phases incompressibles, c'est-à-dire telles que $A_{k}^{v}=0$, la contrainte (5) s'écrit simplement :

$$
\frac{\partial}{\partial x}\left(\alpha_{1}\left\langle u_{1}\right\rangle_{1}^{\mathcal{R}}+\alpha_{2}\left\langle u_{2}\right\rangle_{2}^{\mathcal{R}}\right)=0
$$

\section{Eléments de modélisation des volumes}

La contrainte de conservation des volumes énoncée à la section 4.2 sert de guide pour la modélisation des volumes qui constitue une étape importante dans la construction d'un modèle. Celle-ci permet notamment 
d'éclairer le contenu physique de certains modèles de champs eulériens actuellement utilisés ou étudiés, et de pouvoir établir un lien entre une description microscopique instantanée et les modèles d'EDP qui en découlent. Cette section a donc pour but d'illustrer le formalisme de modélisation développé dans les sections précédentes, en se focalisant sur les termes de modélisation des volumes. A titre d'exmple, un modèle simple complet pour la section 5.1 est disponnible en annexe.

La section 5.1 propose une modélisation des volumes dans le cas d'écoulements de bulles dispersées dans un liquide. L'accent est donc mis sur les termes de modélisation $A_{k}^{v}$ et $B_{k}^{v}=0$, ainsi que sur la forme prise par la deuxième équation du système (6). Puis, dans la section 5.2 , quelques éléments de réflexion concernant le modèle bifluide standard sont proposés.

Dans ce qui suit, la phase des bulles sera représentée par la phase 1, tandis que la phase 2 correspondra à la phase liquide. De plus, on choisit de simplifier l'EDS sur les volumes en prenant $B_{k}^{v}$. Pour cette section, on se place dans l'espace tri-dimensionnel. En conséquence, une grandeur notée en gras désigne un vecteur et les opérateurs $\nabla_{\boldsymbol{x}} \cdot(\boldsymbol{\Phi}(t, \boldsymbol{x}))$ et $\nabla_{\boldsymbol{x}}(\phi(t, \boldsymbol{x}))$ sont respectivement la divergence et le gradient sur l'espace physique du champ vectoriel $\boldsymbol{\Phi}$ et du champ scalaire $\phi$.

\subsection{Prise en compte d'une équation de type Rayleigh-Plesset simplifiée}

Dans ce premier cas, on ne fait pas l'hypothèse d'équilibre des pressions, c'est-à-dire que l'on ne fait pas l'hypothèse qu'elles sont égales en tout point $\boldsymbol{x}$ et à tout instant $t$. La variation de volume d'une bulle est alors prise en compte à travers deux aspects : (i) la divergence du champ moyen de vitesse de la phase bulle et (ii) l'écart de pression avec la phase liquide. De plus, on fait l'hypothèse que ces deux phénomènes s'additionnent pour donner la variation de volume globale de la bulle.

La variation du volume d'un élément matériel de fluide associée à la divergence du champ de vitesse du fluide est un aspect classique pour les écoulements incompressibles. C'est d'ailleurs de cette propriété qu'est issue la contrainte stationnaire de divergence nulle pour le système des équations de Navier-Stokes incompressible. On choisit donc de modéliser cet effet (i) sur la variation de volume de la bulle de la même façon :

$$
d v_{1}=v_{1} \nabla_{\boldsymbol{x}} \cdot\left(\left\langle\boldsymbol{u}_{1}\right\rangle_{1}^{\mathcal{R}}\right) d t+\mathcal{F} d t
$$

où $\mathcal{F}$ désigne le deuxième effet (ii), qui est associé à l'écart de pression. Dans cette expression, seule la divergence du champs de vitesse moyen en $\boldsymbol{x}$ est retenue, et non pas sa valeur instantanée. Ce choix est cohérent avec l'hypothèse $B_{1}^{v}=0$. D'autres choix sont évidemment possibles. Par exemple, on pourrait supposer que le terme $B_{1}^{v}$ est non nul pour représenter la divergence des fluctuations de vitesse $\left(\boldsymbol{u}_{\mathbf{1}}-\right.$ $\left\langle\boldsymbol{u}_{1}\right\rangle_{1}^{\mathcal{R}}$ ). Pour expliciter le terme $\mathcal{F}$, on admet qu'en présence d'un champs de vitesse moyen $\left\langle\boldsymbol{u}_{1}\right\rangle_{1}^{\mathcal{R}}$ uniforme, les variations du volume de chaque particule de la phase bulle sont régies par une loi issue d'une simplification de l'équation de Rayleigh-Plesset.

Cette loi de pulsation d'une bulle sphérique est établie à partir de l'équation de Rayleigh-Plesset. Celleci décrit les pulsations d'une bulle sphérique dans un champ fluide incompressible infini, on admettra néanmoins qu'elle reste valide dans notre cadre. En négligeant les effets de la viscosité et de la tension de surface, l'équation de Rayleigh-Plesset s'écrit en fonction du rayon $r_{B}$ d'une bulle :

$$
r_{B} \frac{d^{2} r_{B}}{d t^{2}}+\frac{3}{2}\left(\frac{d r_{B}}{d t}\right)^{2}=\frac{p_{B}-p_{L}}{\rho^{0}},
$$

où $p_{B}$ et $p_{L}$ sont les pressions au sein de la bulle et dans le liquide, et $\rho^{0}$ est une densité liquide de référence. On s'intéresse à des situations où la bulle est proche d'un état d'équilibre et faiblement perturbée, $\left|d r_{B} / d t\right| \ll 1$. Par conséquent, on néglige le deuxième terme au membre de gauche et on explicite le premier rayon $r_{B}$ dans le premier terme de l'équation (7). La vitesse $d r_{B} / d t$ étant supposées faibles, on intègre alors cette équation et on trouve une équation de la forme : 


$$
\frac{d r_{B}}{d t}=\tau_{p} \frac{p_{B}-p_{L}}{\rho^{0} r_{B}^{0}},
$$

où $\tau_{p}$ et un temps caractéristique. Les bulles étant sphériques, une équation équivalente portant sur les volumes des bulles peut aisément être déduite de (8).

Cette loi simplifiée fournit alors une expression pour le terme $\mathcal{F}$, et les volumes des bulles sont alors modélisés par l'EDS :

$$
d v_{1}=v_{1} \nabla_{\boldsymbol{x}} \cdot\left(\left\langle\boldsymbol{u}_{1}\right\rangle_{1}^{\mathcal{R}}\right) d t+4 \Pi\left(\frac{3 v_{1}}{4 \Pi}\right)^{2 / 3} \tau_{p} \frac{p_{1}-p_{2}}{\rho^{0} r_{B}^{0}} d t
$$

ce qui fournit le terme de modélisation $A_{1}^{v}$ suivant :

$$
A_{1}^{v}=v_{1} \nabla_{\boldsymbol{x}} \cdot\left(\left\langle\boldsymbol{u}_{1}\right\rangle_{1}^{\mathcal{R}}\right)+4 \Pi\left(\frac{3 v_{1}}{4 \Pi}\right)^{2 / 3} \tau_{p} \frac{p_{1}-p_{2}}{\rho^{0} r_{B}^{0}} .
$$

Le choix du terme de modélisation des volumes des particules liquides $A_{2}^{v}$ doit être fait tel que, étant donné le terme $A_{1}^{v}$ choisi, la contrainte de conservation du volume (5) soit respectée. Cette contrainte portant sur des valeurs moyennes et le terme $A_{2}^{v}$ étant instantané, le choix n'est donc pas unique. En pratique, lorsque l'on s'intéresse à des écoulements de bulles dispersées dans un liquide comme c'est le cas ici, la forme instantanée exacte de $A_{2}^{v}$ est secondaire. On peut donc se contenter de choisir par exemple :

$$
\alpha_{2} \frac{A_{2}^{v}}{v_{2}}=\nabla_{\boldsymbol{x}} \cdot\left(\alpha_{1}\left\langle u_{1}\right\rangle_{1}^{\mathcal{R}}+\alpha_{2}\left\langle u_{2}\right\rangle_{2}^{\mathcal{R}}\right)-\alpha_{1}\left\langle\frac{A_{1}^{v}}{v_{1}} \mid Z_{1}^{E}\right\rangle_{1}^{\mathcal{R}} .
$$

Une fois les termes $A_{1}^{v}$ et $A_{2}^{v}$ précisés, l'étape de modélisation des volumes est terminée. Avec les choix présentés ci-dessus et en omettant le terme associé au changement de phase, le sous-système (6) s'écrit :

$$
\left\{\begin{array}{l}
\alpha_{1}(t, \boldsymbol{x})+\alpha_{2}(t, \boldsymbol{x})=1, \\
\frac{\partial}{\partial t}\left(\alpha_{1}\right)+\left\langle\boldsymbol{u}_{1}\right\rangle_{1}^{\mathcal{R}} \nabla_{\boldsymbol{x}}\left(\alpha_{1}\right)=\alpha_{1} \frac{(36 \Pi)^{1 / 3}}{\rho^{0} r_{B}^{0}}\left\langle\tau_{p}\left(p_{1}-p_{2}\right) v_{1}^{-1 / 3} \mid Z_{1}^{E}\right\rangle_{1}^{\mathcal{R}} .
\end{array}\right.
$$

L'élément le plus important dans le terme du second membre de la deuxième équation de (10) est le retour à l'équilibre des pressions. La forme de cette équation est très proche de celle qui apparaît dans les modèles bifluides avec relaxation de pressions. Ceux-ci ont notamment été décrits dans $[7,8]$ pour les écoulements de type eau-vapeur et dans $[9,10]$ pour des écoulements gaz-particules sur des applications en détonique. Un modèle stochastique bifluide à relaxation de pressions complet est décrit en annexe.

Le modèle simplifié (8) pour la pulsation de la bulle est d'ordre 1 en temps, tandis que le modèle de Rayleigh-Plesset est d'ordre 2. En ajoutant au modèle la variable $W_{1}$ qui décrit la vitesse de variation du rayon de la bulle, on peut tout à fait intégrer (7). Le modèle qui en découle est alors proche de celui proposé dans [11].

\subsection{Eléments de réflexion concernant le modèle bifluide standard}

Dans la section 5.1, une équation sur les champs de fraction volumique a été déduite à partir d'une modélisation locale instantanée des volumes des particules (i.e. le choix des termes $A_{1}^{v}$ et $A_{2}^{v}$ ). Le problème va maintenant être considéré avec le point de vue inverse : étant donné un système d'EDP sur des champs moyens, quelles sont les formes admissibles pour les termes de modélisation $A_{1}^{v}$ et $A_{2}^{v}$ ? En effet, les EDP ne peuvent fournir des indications que sur les moyennes des termes de modélisation des EDS, la forme instantanée de ces derniers ne peut donc généralement pas être définie de manière unique à partir des EDP. De plus, les modèles sur champs moyens sont souvent écrits en omettant les opérateurs de 
moyennes statistiques, ce qui peut être à l'origine d'ambiguïtés dans l'identification de certains termes. En conséquence, on se place dans un cadre déterministe, c'est-à-dire que pour toute grandeur $\phi_{k}$ on a : $\phi_{k}=\left\langle\phi_{k}\right\rangle_{k}^{\mathcal{F}}=\left\langle\phi_{k}\right\rangle_{k}^{\mathcal{R}}$.

On va s'intéresser au modèle sur champs moyens bifluide standard [1,2], et plus particulièrement à sa partie convective (i.e on ne garde que les termes contenant des dérivées d'ordre 1 en espace ou en temps), en négligeant les effets de masse ajoutée. Contrairement au modèle de la section précédente, ce modèle fait l'hypothèse que les pressions des deux phases sont égales à chaque instant et en tout point de l'écoulement. Dans son écriture la plus classique, il est composé de deux équations de conservation des masses partielles moyennes, deux équations sur les moments phasiques et deux équations sur les énergies totales :

$$
\left\{\begin{array}{l}
\frac{\partial}{\partial t}\left(\alpha_{k} \rho_{k}\right)+\nabla_{\boldsymbol{x}} \cdot\left(\alpha_{k} \rho_{k} \boldsymbol{u}_{k}\right)=0, \quad k=1,2, \\
\frac{\partial}{\partial t}\left(\alpha_{k} \rho_{k} \boldsymbol{u}_{k}\right)+\nabla_{\boldsymbol{x}} \cdot\left(\alpha_{k} \rho_{k} \boldsymbol{u}_{k} \otimes \boldsymbol{u}_{k}+\alpha_{k} p \mathcal{I}_{3}\right)-p \nabla_{\boldsymbol{x}}\left(\alpha_{k}\right)=0, \quad k=1,2, \\
\frac{\partial}{\partial t}\left(\alpha_{k} \rho_{k} E_{k}\right)+\nabla_{\boldsymbol{x}} \cdot\left(\alpha_{k} \boldsymbol{u}_{k}\left(\rho_{k} E_{k}+p\right)\right)+p \frac{\partial}{\partial t}\left(\alpha_{k}\right)=0, \quad k=1,2,
\end{array}\right.
$$

où $E_{k}$ désigne l'énergie totale phasique : $E_{k}=e_{k}+\boldsymbol{u}_{k}^{2} / 2$ et $\mathcal{I}_{3}$ la matrice identité. Ce système d'EDP est complété par la relation $\alpha_{1}+\alpha_{2}=1$.

L'équation d'évolution sur la fraction volumique $\alpha_{1}$ n'apparaît donc pas dans le système, qui ne contient que des informations massiques. Néanmoins, les deux équations portant sur l'énergie se ré-écrivent sous forme d'une équation sur la pression et d'une équation sur $\alpha_{1}$. Cette dernière s'écrit alors :

$$
\begin{array}{r}
\frac{\partial}{\partial t}\left(\alpha_{1}\right)+\frac{a_{1} \boldsymbol{u}_{1}+a_{2} \boldsymbol{u}_{2}}{a_{1}+a_{2}} \nabla_{\boldsymbol{x}}\left(\alpha_{1}\right)+\frac{\boldsymbol{u}_{1}-\boldsymbol{u}_{2}}{a_{1}+a_{2}} \nabla_{\boldsymbol{x}}(p) \\
+\frac{\alpha_{1} a_{1}}{a_{1}+a_{2}} \nabla_{\boldsymbol{x}} \cdot\left(\boldsymbol{u}_{1}\right)-\frac{\alpha_{2} a_{2}}{a_{1}+a_{2}} \nabla_{\boldsymbol{x}} \cdot\left(\boldsymbol{u}_{2}\right)=0
\end{array}
$$

avec $\alpha_{1} a_{1}=\rho_{1} C_{1}^{2}$ et $\alpha_{2} a_{2}=\rho_{2} C_{2}^{2}$, où $C_{1}$ et $C_{2}$ désignent les vitesses du son :

$$
\rho_{k} C_{k}^{2}=\left(\frac{\partial}{\partial p}\left(e_{k}\right)\right)^{-1}\left(\frac{p}{\rho_{k}}-\rho_{k} \frac{\partial}{\partial \rho_{k}}\left(e_{k}\right)\right) \text {. }
$$

Les formes admissibles du terme de modélisation des volumes $A_{1}^{v}$ que l'on peut déduire en identifiant les équations (11) et (6) (sans le terme de changement de phase et en omettant les opérateurs de moyenne) sont complexes :

$$
\begin{array}{r}
\alpha_{1} \frac{A_{1}^{v}}{v_{1}}=\nabla_{\boldsymbol{x}} \cdot\left(\alpha_{1} \boldsymbol{u}_{1}\right)- \\
-\frac{a_{1} \boldsymbol{u}_{1}+a_{2} \boldsymbol{u}_{2}}{a_{1}+a_{2}} \nabla_{\boldsymbol{x}}\left(\alpha_{1}\right)-\frac{\boldsymbol{u}_{1}-\boldsymbol{u}_{2}}{a_{1}+a_{2}} \nabla_{\boldsymbol{x}}(p) \\
-\frac{\alpha_{1} a_{1}}{a_{1}+a_{2}} \nabla_{\boldsymbol{x}} \cdot\left(\boldsymbol{u}_{1}\right)+\frac{\alpha_{2} a_{2}}{a_{1}+a_{2}} \nabla_{\boldsymbol{x}} \cdot\left(\boldsymbol{u}_{2}\right) .
\end{array}
$$

Ce terme permet de définir la loi de variation en temps du volume d'une bulle au sens du modèle bifluide standard. S'il est donc possible de trouver une expression pour $A_{1}^{v}$, sa formulation apparaît comme compliquée et, de plus, son interprétation physique reste une question ouverte.

\section{Conclusions}

Les bases d'un formalisme stochastique de modélisation des écoulements diphasiques compressibles avec changement de phase ont été exposées. Cette modélisation est faite à partir d'une description particulaire lagrangienne de l'écoulement, en utilisant des lois de comportement instantanées. A partir de cette description sous forme d'EDS, la forme générale des EDP portant sur les champs moyens eulériens des fluides 
a été explicitée. Ceci a permis de clarifier la notion de conservation du volume au sens de la description retenue. Les contraintes sur les lois de comportement assurant le respect de cette conservation ont été écrites. Enfin, des éléments concrets de modélisation ont permis d'illustrer la démarche.

L'intérêt de la démarche qui a été développée dans cet article est de proposer un lien entre une physique microscopique (écrite sur les variables instantanées associées aux particules) et les modèles eulériens (portant sur des champs moyens) qui en résultent. Cette approche permet ainsi de mieux aborder des questions précises, notamment la contrainte de conservation du volume, et de pouvoir mieux éclairer le contenu physique des modèles eulériens macroscopiques.

Dans la dernière partie de ce document, essentiellement par souci de concision, l'approche a été appliquée partiellement. Ainsi, l'accent a été mis sur la modélisation des volumes des particules. Dans le cas de bulles dispersées, le premier exemple a permis d'illustrer le passage d'une équation issue de l'équation de Rayleigh-Plesset, à une équation sur le champ fraction volumique. Cette dernière prend une forme proche de celle qui apparaît dans certains modèles bifluides de la littérature. Il est en fait possible, sous certaines conditions, de trouver un modèle d'EDS complet et physiquement admissible dont les champs moyens sont régis par les mêmes EDP que ces modèles bifluides. Dans la section 5.2, les éléments proposés concernant le modèle bifluide standard font apparaître une description assez complexe des variations de volume instantanées des bulles.

En ce qui concerne le changement de phase, une modélisation locale a été proposée. En revanche, les termes sources associés dans les EDP ne semble pas pouvoir être explicités en fonction des grandeurs moyennes eulériennes. Il est tout à fait envisageable d'utiliser ce modèle tel qu'il est décrit pour la simulation numérique. Pour ce faire, il est possible de construire une méthode mélangeant les systèmes d'EDS et d'EDP en suivant l'esprit des méthodes hybrides comme celle présentée dans [12] ou [13]. Cette dernière méthode combine une approche par champs eulériens et une approche particulaire lagrangienne, et son but est de permettre une meilleure prise en compte de la turbulence dans le cadre d'écoulements de type gaz-particules. En conservant la même idée, on peut construire un schéma numérique à pas fractionnaires dont la première étape consiste à discrétiser les trois premiers termes de l'équation (6) par une technique adaptée aux EDP; la deuxième étape correspond à la prise en compte du changement de phase tel qu'il est décrit en section 2.3. Une telle méthode permet alors de prendre en compte le changement de phase de façon locale sans imposer de fermeture dépendant des champs moyens eulériens.

\section{Références}

[1] M. Ishii, T. Hibiki, Thermo-fluid dynamics of two phase flow, Springer (2005).

[2] H. B. Stewart, B. Wendroff, Two-Phase flow : models and methods, J. Comp. Phys., 56 (1984) $363-409$.

[3] S. B. Pope, Turbulent flows, Cambridge University Press, 2000.

[4] J.-P. Minier, E. Peirano, The pdf approach to turbulent polydispersed two-phase flows, Phys. Rep., 352 (2001) 1-214.

[5] B. J. Delarue, S. B. Pope, Application of PDF methods to compressible turbulent flows, Phys. Fluids, 9 (1997) 27042715 .

[6] C. Gardiner, Handbook of stochastic methods for physics, chemistry and natural sciences, Springer (1985).

[7] F. Coquel, T. Gallouët, J.-M. Hérard, N. Seguin, Closure laws for a two-fluid two-pressure model, C.R. Acad. Sci. Paris, I-334 (2002) 927-932.

[8] V. Ransom, D. Hicks, Hyperbolic two-pressure model for two-phase flow, J. of Comp. Phys., 53 (1984) $124-151$.

[9] M. R. Baer, J. W. Nunziato, A two phase miwture theory for the deflagration-to-detonation transition (ddt) in reactive granular materials, Int. J. for Multiphase Flow, 12-6 (1986) 861-889.

[10] A. K. Kapila, S. F. Son, J. B. Bdzil, R. Menikoff, D. S. Stewart, Two-phase modeling of ddt : structure of the velocity relaxation zone, Phys. of Fluids, 9 (1997) 3885-3897. 
[11] S. Gavrilyuk, R. Saurel, Mathematical and Numerical Modeling of Two-Phase Compressible Flows with Micro-Inertia, J. of Comp. Phys., 175-1 (2002) 326-360.

[12] M. Muradoglu,P. Jenny,S.B. Pope,D.A. Caughey, A consistent hybrid finite-volume/particle method for the pdf equations of turbulent reactive flows, J. Comp. Phys., 154 (1999) 342-371.

[13] K. Dorogan, J.-M. Hérard, J.-P. Minier, A relaxation scheme for hybrid modelling of gas-particle flows, submitted to publication.

\section{Annexe : un modèle simple pour les écoulements dispersés eau-vapeur}

Cette annexe présente un modèle stochastique lagrangien pour les écoulements diphasiques dispersés eau-vapeur. Il correspond à une description assez simple de ces écoulements, sans échange avec l'extérieur. La turbulence est isotrope instationnaire et ne revêt qu'un aspect dynamique (et pas thermodynamique). D'autre part, la loi de fermeture du terme $\mathcal{F}$, décrivant les pulsations d'une bulle, est différente de celle proposée dans la section 5.1. Ce choix permet de retrouver un ensemble d'EDP proche de celui du modèle bifluide à relaxation de pression, dont les caractéristiques sont décrites dans $[7,8,9,10]$. Les termes de modélisation des deux phases sont choisis conjointement, conformément à la contrainte de conservation du volume (voir section 4.2) et de manière à ce que la masse moyenne du mélange, le moment moyen du mélange et l'énergie totale moyenne du mélange soient conservées (en l'absence de sources externes, le mélange des deux phases constitue un système fermé).

\section{Modélisation des volumes.}

Les termes de modélisation des volumes sont : $B_{1}^{v}=B_{2}^{v}=0$, et

$$
A_{1}^{v}=v_{1} \nabla_{\boldsymbol{x}} \cdot\left(\left\langle\boldsymbol{u}_{\mathbf{1}}\right\rangle_{1}^{\mathcal{R}}\right)+\frac{v_{1}}{K_{p}}\left(\left\langle p_{1}\right\rangle_{1}^{\mathcal{R}}-\left\langle p_{2}\right\rangle_{2}^{\mathcal{R}}\right)
$$

La grandeur $K_{p}$ est une constante statistique positive, par exemple :

$$
\frac{1}{K_{p}}=\frac{1}{\tau_{p}} \frac{\alpha_{2}}{\left|\left\langle p_{1}\right\rangle_{1}^{\mathcal{R}}\right|+\left|\left\langle p_{2}\right\rangle_{2}^{\mathcal{R}}\right|}
$$

où $\tau_{p}$ est une constante de temps positive. Afin d'assurer la conservation du volume, on choisit :

$$
\frac{\alpha_{2}}{v_{2}} A_{2}^{v}=\left(\alpha_{2} \nabla_{\boldsymbol{x}} \cdot\left(\left\langle\boldsymbol{u}_{\mathbf{2}}\right\rangle_{2}^{\mathcal{R}}\right)-\alpha_{1}\left\langle\frac{\mathcal{F}}{v_{1}}\right\rangle_{1}^{\mathcal{R}}\right)+\left(\left\langle\boldsymbol{u}_{\mathbf{1}}\right\rangle_{1}^{\mathcal{R}}-\left\langle\boldsymbol{u}_{\mathbf{2}}\right\rangle_{2}^{\mathcal{R}}\right) \cdot \nabla_{\boldsymbol{x}}\left(\alpha_{1}\right) .
$$

\section{Modélisation des vitesses.}

Les termes de modélisation des vitesses sont :

$$
\alpha_{2} \rho_{2} \boldsymbol{A}_{2}^{u}=-\alpha_{2} \nabla_{\boldsymbol{x}}\left(\left\langle p_{2}\right\rangle_{2}^{\mathcal{R}}\right)+\mathcal{D}_{2}+\frac{1}{T_{2}^{L}} \frac{\alpha_{2} \rho_{2} \alpha_{1}\left\langle\rho_{1}\right\rangle_{1}^{\mathcal{R}}}{\alpha_{1}\left\langle\rho_{1}\right\rangle_{1}^{\mathcal{R}}+\alpha_{2}\left\langle\rho_{2}\right\rangle_{2}^{\mathcal{R}}}\left(\left\langle\boldsymbol{u}_{\mathbf{2}}\right\rangle_{2}^{\mathcal{F}}-\boldsymbol{u}_{\mathbf{2}}\right)
$$

où $\mathcal{D}_{2}$ est la force de trainée statique et s'écrit :

$$
\mathcal{D}_{2}=\frac{1}{\tau_{u}} \frac{\alpha_{2} \rho_{2} \alpha_{1}\left\langle\rho_{1}\right\rangle_{1}^{\mathcal{R}}}{\alpha_{1}\left\langle\rho_{1}\right\rangle_{1}^{\mathcal{R}}+\alpha_{2}\left\langle\rho_{2}\right\rangle_{2}^{\mathcal{R}}}\left(\left\langle\boldsymbol{u}_{\mathbf{1}}\right\rangle_{1}^{\mathcal{F}}-\boldsymbol{u}_{\mathbf{2}}\right)
$$

Les constantes de temps $\tau_{u}$ et $T_{2}^{L}$ sont des constantes statistiques positives. Pour la phase bulle, on choisit : 


$$
\begin{aligned}
\alpha_{1} \rho_{1} \boldsymbol{A}_{1}^{u}=-\alpha_{1} \nabla_{\boldsymbol{x}} & \left(\left\langle p_{1}\right\rangle_{1}^{\mathcal{R}}\right)+\mathcal{D}_{1}+\left(\left\langle p_{2}\right\rangle_{2}^{\mathcal{R}}-\left\langle p_{1}\right\rangle_{1}^{\mathcal{R}}\right) \nabla_{\boldsymbol{x}}\left(\alpha_{1}\right) \\
& +\frac{1}{T_{1}^{L}} \frac{\alpha_{1} \rho_{1} \alpha_{2}\left\langle\rho_{2}\right\rangle_{2}^{\mathcal{R}}}{\alpha_{1}\left\langle\rho_{1}\right\rangle_{1}^{\mathcal{R}}+\alpha_{2}\left\langle\rho_{2}\right\rangle_{2}^{\mathcal{R}}}\left(\left\langle\boldsymbol{u}_{1}\right\rangle_{1}^{\mathcal{F}}-\boldsymbol{u}_{\mathbf{1}}\right)
\end{aligned}
$$

où $T_{1}^{L}$ est une constante statistique positive et où :

$$
\mathcal{D}_{1}=\frac{1}{\tau_{u}} \frac{\alpha_{1} \rho_{1} \alpha_{2}\left\langle\rho_{2}\right\rangle_{2}^{\mathcal{R}}}{\alpha_{1}\left\langle\rho_{1}\right\rangle_{1}^{\mathcal{R}}+\alpha_{2}\left\langle\rho_{2}\right\rangle_{2}^{\mathcal{R}}}\left(\left\langle\boldsymbol{u}_{2}\right\rangle_{2}^{\mathcal{F}}-\boldsymbol{u}_{1}\right)
$$

En supposant que les termes de dispersion des vitesses sont isotropes, $\boldsymbol{B}_{k}^{u}=B_{k}^{u}(1,1,1)^{\top}$, on choisit :

$$
\alpha_{k}\left\langle\rho_{k}\right\rangle_{k}^{\mathcal{R}}\left(B_{k}^{u}\right)^{2}=\frac{4}{3} \frac{\alpha_{1}\left\langle\rho_{1}\right\rangle_{1}^{\mathcal{R}} \alpha_{2}\left\langle\rho_{2}\right\rangle_{2}^{\mathcal{R}}}{\alpha_{1}\left\langle\rho_{1}\right\rangle_{1}^{\mathcal{R}}+\alpha_{2}\left\langle\rho_{2}\right\rangle_{2}^{\mathcal{R}}}\left(\frac{1}{\tau_{u}}+\frac{1}{T_{k}^{L}}\right) e_{k}^{T}-\alpha_{k}\left\langle\rho_{k}\right\rangle_{k}^{\mathcal{R}} \frac{2\left\langle\epsilon_{k}\right\rangle_{k}^{\mathcal{F}}}{3}
$$

En considérant la constante statistique positive $\tau_{\epsilon, k}$ telle que :

$$
\frac{1}{\tau_{\epsilon, k}}<2\left(\frac{1}{\tau_{u}}+\frac{1}{T_{k}^{L}}\right)
$$

la dissipation $\epsilon_{k}$ est modélisée comme suit :

$$
\alpha_{k}\left\langle\rho_{k}\right\rangle_{k}^{\mathcal{R}}\left\langle\epsilon_{k}\right\rangle_{k}^{\mathcal{F}}=\frac{\alpha_{1}\left\langle\rho_{1}\right\rangle_{1}^{\mathcal{R}} \alpha_{2}\left\langle\rho_{2}\right\rangle_{2}^{\mathcal{R}}}{\alpha_{1}\left\langle\rho_{1}\right\rangle_{1}^{\mathcal{R}}+\alpha_{2}\left\langle\rho_{2}\right\rangle_{2}^{\mathcal{R}}} \frac{e_{k}^{T}}{\tau_{\epsilon, k}}
$$

où $e_{k}^{T}$ est l'énergie cinétique turbulente :

$$
e_{k}^{T}=\frac{1}{2}\left\langle\left(\left\langle\boldsymbol{u}_{\boldsymbol{k}}\right\rangle_{k}^{\mathcal{F}}-\boldsymbol{u}_{\boldsymbol{k}}\right) \cdot\left(\left\langle\boldsymbol{u}_{\boldsymbol{k}}\right\rangle_{k}^{\mathcal{F}}-\boldsymbol{u}_{\boldsymbol{k}}\right)\right\rangle_{k}^{\mathcal{F}} .
$$

\section{Modélisation des énergies internes.}

Les énergies internes sont modélisées conformément au premier principe de la thermodynamique. Les termes de dispersion sont nuls, $B_{k}^{e}=0$, et les termes de drift sont :

$$
\alpha_{k} \rho_{k} A_{k}^{e}=-\alpha_{k} p_{k} \frac{A_{k}^{v}}{v_{k}}+F_{k}+I_{k}+\alpha_{k}\left\langle\rho_{k}\right\rangle_{k}^{\mathcal{R}}\left\langle\epsilon_{k}\right\rangle_{k}^{\mathcal{F}} \quad \text { et } \quad B_{k}^{e}=0 .
$$

Les différents termes sont :

$$
\begin{gathered}
F_{1}=\frac{\left\langle\boldsymbol{u}_{2}\right\rangle_{2}^{\mathcal{F}}-\left\langle\boldsymbol{u}_{1}\right\rangle_{1}^{\mathcal{F}}}{2} \cdot \mathcal{D}_{1} \quad \text { et } \quad F_{2}=\frac{\left\langle\boldsymbol{u}_{1}\right\rangle_{1}^{\mathcal{F}}-\left\langle\boldsymbol{u}_{2}\right\rangle_{2}^{\mathcal{F}}}{2} \cdot \mathcal{D}_{2}, \\
I_{2}=0 \text { et } I_{1}=\alpha_{1} \frac{\mathcal{F}}{v_{1}}\left(\left\langle p_{2}\right\rangle_{2}^{\mathcal{R}}-p_{1}\right) .
\end{gathered}
$$

Modélisation du changement de phase.

La modélisation du changement de phase est telle que décrite dans la section 2.3 et nécessite les définitions des lois d'état des potentiels chimiques, de $\delta t$ et de $\tau$.

Ainsi, pourvu que les lois d'état des pressions $\mathcal{P}_{k}$ et des potentiels chimiques $\mu_{k}$ soient données, et pourvu que les constantes $\tau, \delta t, \tau_{p}, \tau_{u}, T_{k}^{L}, \tau_{\epsilon, k}$ soient déterminées, le modèle stochastique est fermé. D'autre part, ce modèle assure la positivité des volumes.

\section{Equations sur les champs moyens.}

On peut déduire de ce modèle stochastique, un ensemble d'EDP portant sur des champs moyens proche 
des modèles à relaxation de pressions [7,8,9,10]. Dans ce qui suit, l'énergie totale $E_{k}$ d'une particule est la somme de son énergie interne et de son énergie cinétique $E_{k}=e_{k}+u_{k}^{2} / 2$. Le système d'équations portant sur les fractions volumiques, les masses partielles, les moments et les énergies totales est :

$$
\begin{aligned}
& \alpha_{1}(t, \boldsymbol{x})+\alpha_{2}(t, \boldsymbol{x})=1, \\
& \frac{\partial}{\partial t}\left(\alpha_{1}\right)+\left\langle\boldsymbol{u}_{1}\right\rangle_{1}^{\mathcal{R}} \cdot \nabla_{\boldsymbol{x}}\left(\alpha_{1}\right)=\alpha_{1}\left\langle\frac{\mathcal{F}}{v_{1}}\right\rangle_{1}^{\mathcal{R}}+\int v_{1}^{*} \delta\left(\boldsymbol{x}-\boldsymbol{x}_{1}^{*}\right) \mathcal{T}_{1}\left(t ; Z_{1}^{L}\right) d Z_{1}^{L}, \\
& \frac{\partial}{\partial t}\left(\alpha_{1}\left\langle\rho_{1}\right\rangle_{1}^{\mathcal{R}}\right)+\nabla_{\boldsymbol{x}} \cdot\left(\alpha_{1}\left\langle\rho_{1}\right\rangle_{1}^{\mathcal{R}}\left\langle\boldsymbol{u}_{1}\right\rangle_{1}^{\mathcal{F}}\right)=\int m_{1}^{*} \delta\left(\boldsymbol{x}-\boldsymbol{x}_{1}^{*}\right) \mathcal{T}_{1}\left(t ; Z_{1}^{L}\right) d Z_{1}^{L}, \\
& \frac{\partial}{\partial t}\left(\alpha_{2}\left\langle\rho_{2}\right\rangle_{2}^{\mathcal{R}}\right)+\nabla_{\boldsymbol{x}} \cdot\left(\alpha_{2}\left\langle\rho_{2}\right\rangle_{2}^{\mathcal{R}}\left\langle\boldsymbol{u}_{2}\right\rangle_{2}^{\mathcal{F}}\right)=\int m_{2}^{*} \delta\left(\boldsymbol{x}-\boldsymbol{x}_{2}^{*}\right) \mathcal{T}_{2}\left(t ; Z_{2}^{L}\right) d Z_{2}^{L}, \\
& \frac{\partial}{\partial t}\left(\alpha_{1}\left\langle\rho_{1}\right\rangle_{1}^{\mathcal{R}}\left\langle\boldsymbol{u}_{1}\right\rangle_{1}^{\mathcal{F}}\right)+\nabla_{\boldsymbol{x}} \cdot\left(\alpha_{1}\left\langle\rho_{1}\right\rangle_{1}^{\mathcal{R}}\left\langle\boldsymbol{u}_{1} \otimes \boldsymbol{u}_{1}\right\rangle_{1}^{\mathcal{F}}+\alpha_{1}\left\langle p_{1}\right\rangle_{1}^{\mathcal{R}} \boldsymbol{I}_{3}\right)-\left\langle p_{2}\right\rangle_{2}^{\mathcal{R}} \nabla_{\boldsymbol{x}}\left(\alpha_{1}\right)= \\
& \left\langle\mathcal{D}_{1}\right\rangle_{1}^{\mathcal{R}}+\int m_{1}^{*} \boldsymbol{u}_{1} \delta\left(\boldsymbol{x}-\boldsymbol{x}_{1}^{*}\right) \mathcal{T}_{1}\left(t ; Z_{1}^{L}\right) d Z_{1}^{L}, \\
& \frac{\partial}{\partial t}\left(\alpha_{2}\left\langle\rho_{2}\right\rangle_{2}^{\mathcal{R}}\left\langle\boldsymbol{u}_{2}\right\rangle_{2}^{\mathcal{F}}\right)+\nabla_{\boldsymbol{x}} \cdot\left(\alpha_{2}\left\langle\rho_{2}\right\rangle_{2}^{\mathcal{R}}\left\langle\boldsymbol{u}_{2} \otimes \boldsymbol{u}_{2}\right\rangle_{2}^{\mathcal{F}}+\alpha_{2}\left\langle p_{2}\right\rangle_{2}^{\mathcal{R}} \boldsymbol{I}_{3}\right)-\left\langle p_{2}\right\rangle_{2}^{\mathcal{R}} \nabla_{\boldsymbol{x}}\left(\alpha_{2}\right)= \\
& \left\langle\mathcal{D}_{2}\right\rangle_{2}^{\mathcal{R}}+\int m_{2}^{*} \boldsymbol{u}_{2} \delta\left(\boldsymbol{x}-\boldsymbol{x}_{2}^{*}\right) \mathcal{T}_{2}\left(t ; Z_{2}^{L}\right) d Z_{2}^{L}, \\
& \frac{\partial}{\partial t}\left(\alpha_{1}\left\langle\rho_{1}\right\rangle_{1}^{\mathcal{R}}\left\langle E_{1}\right\rangle_{1}^{\mathcal{F}}\right)+\nabla_{\boldsymbol{x}} \cdot\left(\alpha_{1}\left\langle\rho_{1}\right\rangle_{1}^{\mathcal{R}}\left\langle\boldsymbol{u}_{1} E_{1}\right\rangle_{1}^{\mathcal{F}}+\alpha_{1}\left\langle p_{1}\right\rangle_{1}^{\mathcal{R}}\left\langle\boldsymbol{u}_{1}\right\rangle_{1}^{\mathcal{R}}\right)+\left\langle p_{2}\right\rangle_{2}^{\mathcal{R}} \frac{\partial}{\partial t}\left(\alpha_{1}\right)= \\
& \frac{\left\langle\boldsymbol{u}_{1}\right\rangle_{1}^{\mathcal{F}}+\left\langle\boldsymbol{u}_{2}\right\rangle_{2}^{\mathcal{F}}}{2} \cdot\left\langle\mathcal{D}_{1}\right\rangle_{1}^{\mathcal{R}}+\int m_{1}^{*} E_{1} \delta\left(\boldsymbol{x}-\boldsymbol{x}_{1}^{*}\right) \mathcal{T}_{1}\left(t ; Z_{1}^{L}\right) d Z_{1}^{L}+\left\langle p_{2}\right\rangle_{2}^{\mathcal{R}} \int v_{1}^{*} \delta\left(\boldsymbol{x}-\boldsymbol{x}_{1}^{*}\right) \mathcal{T}_{1}\left(t ; Z_{1}^{L}\right) d Z_{1}^{L}, \\
& \frac{\partial}{\partial t}\left(\alpha_{2}\left\langle\rho_{2}\right\rangle_{2}^{\mathcal{R}}\left\langle E_{2}\right\rangle_{2}^{\mathcal{F}}\right)+\nabla_{\boldsymbol{x}} \cdot\left(\alpha_{2}\left\langle\rho_{2}\right\rangle_{2}^{\mathcal{R}}\left\langle\boldsymbol{u}_{2} E_{2}\right\rangle_{2}^{\mathcal{F}}+\alpha_{2}\left\langle p_{2}\right\rangle_{2}^{\mathcal{R}}\left\langle\boldsymbol{u}_{2}\right\rangle_{2}^{\mathcal{R}}\right)+\left\langle p_{2}\right\rangle_{2}^{\mathcal{R}} \frac{\partial}{\partial t}\left(\alpha_{2}\right)= \\
& \frac{\left\langle\boldsymbol{u}_{1}\right\rangle_{1}^{\mathcal{F}}+\left\langle\boldsymbol{u}_{2}\right\rangle_{2}^{\mathcal{F}}}{2} \cdot\left\langle\mathcal{D}_{2}\right\rangle_{2}^{\mathcal{R}}+\int m_{2}^{*} E_{2} \delta\left(\boldsymbol{x}-\boldsymbol{x}_{2}^{*}\right) \mathcal{T}_{2}\left(t ; Z_{2}^{L}\right) d Z_{2}^{L}+\left\langle p_{2}\right\rangle_{2}^{\mathcal{R}} \int v_{2}^{*} \delta\left(\boldsymbol{x}-\boldsymbol{x}_{2}^{*}\right) \mathcal{T}_{2}\left(t ; Z_{2}^{L}\right) d Z_{2}^{L} .
\end{aligned}
$$

La matrice identité de taille 3 est notée $\boldsymbol{I}_{3}$ et $\otimes$ désigne le produit tensoriel de deux vecteurs.

Ce modèle assure donc la conservation de la masse moyenne totale, du moment moyen total et de l'énergie totale moyenne du mélange. Il est important de noter que cet ensemble de huit équations, considéré indépendamment du modèle stochastique, n'est pas fermé. 